\title{
Impact of living donor liver with steatosis and idiopathic portal inflammation on clinical outcomes in pediatric liver transplantation: Beijing experience
}

\author{
Xinyan Zhao ${ }^{1,2,3}$, Yafei $\mathrm{He}^{2,3,4 \#}$, Jimin Liu ${ }^{5}$, Qian Zhang ${ }^{3,6}$, Liwei Liu ${ }^{1,2,3}$, Wei Qu ${ }^{2,3,4}$, Ying Liu ${ }^{2,3,4}$, \\ Zhigui Zeng ${ }^{2,3,4}$, Haiming Zhang ${ }^{2,3,4}$, Jidong Jia ${ }^{1,2,3}$, Liying Sun ${ }^{2,3,4}$, Lin Wei ${ }^{2,3,4}$, Zhijun Zhu ${ }^{2,3,4}$
}

${ }^{1}$ Liver Research Center, Beijing Friendship Hospital, Capital Medical University, Beijing, China; ${ }^{2}$ Liver Transplant Center, Beijing Friendship Hospital, Capital Medical University, Beijing, China; ${ }^{3}$ Clinical Center for Pediatric Liver Transplantation, Capital Medical University, Beijing, China; ${ }^{4}$ National Clinical Research Center for Digestive Disease, Beijing, China; ${ }^{5}$ Department of Pathology and Molecular Medicine, Faculty of Health Sciences, McMaster University, Hamilton, ON, Canada; ${ }^{6}$ Clinical Epidemiology and Evidence Base Medicine Unit, Beijing Friendship Hospital, Capital Medical University, Beijing, China

Contributions: (I) Conception and design: Z Zhu, L Sun, L Wei; (II) Administrative support: Z Zhu, L Sun, L Wei; (III) Provision of study materials or patients: Z Zhu, L Sun; (IV) Collection and assembly of data: X Zhao, Y He, L Liu, L Wei, W Qu, Y Liu, Z Zeng, H Zhang; (V) Data analysis and interpretation: Q Zhang, Y He, L Liu; (VI) Manuscript writing: All authors; (VII) Final approval of manuscript: All authors.

\#These authors contributed equally to this work.

Correspondence to: Liying Sun, MD, PhD; Lin Wei, MD, PhD; Zhijun Zhu, MD, PhD. Liver Transplant Center, Beijing Friendship Hospital, Capital Medical University, Clinical Center for Pediatric Liver Transplantation, National Clinical Research Center for Digestive Disease, No. 95 Yong An Road, Xi Cheng District, Beijing, China. Email: sunxlx@outlook.com; 18610932626@163.com; zhu-zhijun@outlook.com.

Background: To evaluate the impact of steatosis and/or idiopathic portal inflammation (IPI) in living donor livers on recipients' clinical outcomes.

Methods: We assessed 305 qualified donor liver samples from June 2013 to December 2018. Donors and recipients' clinical characteristics, including follow-up data were retrieved. The graft and overall survival with/without steatosis or portal inflammation were compared by Kaplan-Meier analysis.

Results: For living donors, the medium age of was $31.2(28,35.8)$ years old; liver histopathology showed macrovesicular steatosis: $0-5 \%$ 264/305 (86.6\%) and 5-30\% 41/305 (13.4\%), IPI: no 220/305 (72.1\%) and mild $85 / 305(27.9 \%)$. For recipients, the medium age was $1.0(0.6,1.5)$ years old; the median pediatric-endstage-liver-disease score was $16(5.0,26.0)$ and medium follow-up time was $32.8(24.8,52.0)$ months. Biliary atresia $(69.5 \%)$ was the main indication for liver transplantation (LT).

Conclusions: The presence of steatosis and portal inflammation of the donor liver did not impact the clinical outcomes including transaminase or bilirubin normalization, short-/long-term complications and recipients' survival. However, recipients with high pediatric-end-stage-liver-disease score (>16) receiving donor liver with portal inflammation, but not steatosis, had trend negative effect on recipients' survival. In conclusion, donor livers with mild steatosis and portal inflammation were qualified for pediatric living donor LT. However, donor liver with mild portal inflammation would better not be allocated to recipients with high pediatric-end-stage-liver-disease score. This study provided new evidence in pediatric living donor liver allocation.

Keywords: Graft/overall survival; idiopathic portal inflammation (IPI); nonalcoholic fatty liver disease (NAFLD); pediatric living donor liver transplantation (LT); steatosis

Submitted Sep 02, 2020. Accepted for publication Dec 24, 2020.

doi: 10.21037/hbsn-20-685

View this article at: http://dx.doi.org/10.21037/hbsn-20-685 


\section{Introduction}

Nonalcoholic fatty liver disease (NAFLD) is a major health burden (1). This burden is expected to increase as incidence of obesity, diabetes mellitus and metabolic syndrome continues to grow. It is particularly relevant to Chinese population since lifestyle has changed dramatically as a result of industrialization and urbanization. The global projected growth of NAFLD is $0-30 \%$, from 2016 to 2030 , with the highest growth in China $(2,3)$. This high prevalence of NAFLD definitely will affect liver transplantation (LT) donor pool.

Living donor LT (LDLT) is a standard of care for end stage liver diseases (4). Pediatric LDLT can be done with excellent results using left liver lobe or left lateral segment (5-7). Assessing the degree of donor hepatic steatosis is one important histological evaluation (8-11). Although mild macrovesicular steatosis (up to $30 \%$ ) does not adversely affect recipients' outcomes in adult whole-liver LTs (11-14) and is also believed to have no adverse effects on clinical outcome of LDLT recipients, there is a need to study this concept in detail in LDLT.

Mild idiopathic portal inflammation (IPI) is another frequently observed feature in 'healthy' living donor with a range from $6.0 \%$ to $16.0 \%(15-18)$. The impact of this finding has also not been reported in $\operatorname{LDLT}(18,19)$. One question of particular interest is the synergism of mild steatosis and mild or mild-moderate IPI's impact on short- and long-term outcomes of pediatric LDLT. This pathologic constellation is crucial for the success of LT especially when dealing with high risk recipients presenting high pediatric end stage liver disease scores (pediatric endstage liver disease, PELD $\geq 16)(20)$. The study aimed at (I) identifying the prevalence of macrovesicular steatosis (5-30\%) and IPI (Ishak >1) in these 'healthy' living donors; (II) evaluating their impact on biochemical evolution, short-/long-term outcome and final outcomes post-LDLT; and (III) evaluating the impact of steatosis or IPI on clinical outcomes in high PELD score patients. We present the following article in accordance with the STROBE reporting checklist (available at https://hbsn.amegroups.com/article/ view/10.21037/hbsn-20-685/rc).

\section{Methods}

Pediatric LDLT recipients (age $<18$-year-old) performed in Beijing Friendship Hospital, Capital Medical University from June 2013 to December 2018 were retrospectively reviewed. Living donor data including age, gender, body mass index (BMI), parameters of liver biochemical tests and imaging results were collected. Abdominal ultrasound and Computed Tomography scan were used routinely for donor assessment. Donors with obvious fatty liver on either imaging study were disqualified as the living donors until a weight loss $8-10 \%$ of baseline level and extent of fatty liver becomes mild degree or less. No donor consumed alcohol $>20 \mathrm{~g} /$ day for female and $>40 \mathrm{~g} /$ day for male in the study.

Recipients' clinical data including baseline demographics, parameters of liver biochemical tests, indications for LDLT were collected from the hospital electronic data base. PELD score was calculated in pediatric patients.

\section{Inclusion and exclusion criteria}

Inclusion criteria were: (I) pediatric recipients defined as age $<18$-year-old. (II) LDLT.

Exclusion criteria were: (I) domino cross LT. (II) Sample size wedge liver specimen was $<0.2 \mathrm{~cm} \times 0.2 \mathrm{~cm}$. (III) Retransplantation.

\section{Living donor liver biopsy processing and histopathological assessment}

The living donor liver wedge biopsy tissues were routinely sampled during living donor liver graft procurement. The living donor liver biopsy were routinely taken at segment 3 (S3) by transplant surgeons in our center. The specimens were fixed in neutral formalin, embedded in paraffin, and routinely stained with hematoxylin and eosin $(\mathrm{H} / \mathrm{E})$ and other stains, including trichrome and reticulin. Corresponding slides were retrieved.

Histological features of the livers from the enrolled cases including steatosis and portal inflammation were systematically assessed by two liver pathologists. Hepatic macrovesicular steatosis was scaled as $0-5 \%$ and $5-30 \%$ steatosis according to the standard criterion (21). Portal inflammation was divided as no, mild and mild to moderate portal inflammation according to Ishak modified histological activity score system (22).

Recipients who received a living donor liver with hepatic macrovesicular steatosis $0-5 \%$ were defined as non-steatosis group, while $5-30 \%$ steatosis was defined as steatosis group. Similarly, recipients who received a living donor liver without portal inflammation were defined as non-IPI group and mild or mild to moderate portal inflammation was defined as IPI group. 


\section{Immunosuppressive management}

Immunosuppressive induction started by methylprednisolone and maintenance therapy was mainly composed of tacrolimus/mycophenolate mofetil with/without low dose prednisolone. In case of tacrolimus intolerance or toxicity, cyclosporin was used.

\section{Follow ups}

Clinical follow up information including serum value of alanine aminotransferase (ALT) and total bilirubin (TBIL) at 1 week, 2 weeks and 4 weeks post LDLTs, short$(\leq 1$-month post LDLT) and long-term complications (>1-month post LDLT), as well as graft/overall survival up to 5 years were retrieved from the clinical database.

\section{Statistics}

Variables were expressed as median (quantile 1-quantile 3) or percentage. The differences of continuous variables were identified by Wilcoxon test. The differences of categorical variables were identified by Chi-square test. In the statistical analysis of the changes in ALT and TBIL before and 1 week, 2 weeks and 4 weeks after LT, the mixed model was used in the repeated measurement of the blood parameters. The 1, 3, 5-year graft/overall survival were assessed by KaplanMeier analysis. Risk factors such as short- and long-term complications associated with the survival were identified using univariate and multivariate Cox proportional hazards regression model. All statistical analyses were performed using SAS software, version 9.4 (SAS Institute Inc., Cary, NC, US). $\mathrm{P}<0.05$ was considered as statistically significant.

\section{Ethical consideration}

The study was conducted in accordance with the Declaration of Helsinki (as revised in 2013). The study was approved by institutional ethics board of Beijing Friendship Hospital (No. 2018-P2-207-01) and informed consent was taken from all individual participants.

\section{Results}

From June 2013 to December 2018, a total of 531 pediatric LTs were performed in Beijing Friendship Hospital. 305 of 358 LDLT cases (85.2\%) were enrolled in the study (Figure 1). Medium follow-up time post LDLT for recipients was 32.8
$(24.8,52.0)$ months.

The median age of donors was $31.2(28.0,35.8)$ years old (Figure $2 A$ ). Male accounted for $42.3 \%$. The median BMI was $22.2(20.1,24.4) \mathrm{kg} / \mathrm{m}^{2}$ (Figure $\left.2 B\right) .176 / 305$ donors' BMI $(57.7 \%)$ was $<23 \mathrm{~kg} / \mathrm{m}^{2}, 69 / 305(22.6 \%)$ between $23-25 \mathrm{~kg} / \mathrm{m}^{2}$ and $60 / 305(19.7 \%)>25 \mathrm{~kg} / \mathrm{m}^{2}$. Median ALT and aspartate aminotransferase were 16.0 (12.0, 24.0; Figure 2C) and $19.6(16.1,23.7)$ IU/L respectively and gamma-glutamyl transpeptidase was $17.0(12.0,24.0) \mathrm{IU} / \mathrm{L}$ (Table 1).

The median age of recipients was $1.0(0.6,1.5)$ years old (Figure $2 D$ ) and male accounted for $51.8 \%$ (Figure $2 E$ ). The indications for LDLT were biliary atresia $(69.5 \%)$ followed by genetic/metabolic liver diseases (23) (23.3\%) or other liver diseases $(7.2 \%$; Figure $2 F)$. The medium PELD score was $16.0(5.0,26.0 ;$ Table 1$)$.

\section{Donor liver histological distribution of hepatic steatosis and IPI}

Macrovesicular steatosis up to $30 \%$ was identified in 144/305 (47.2\%) donors: 0 in 161/305 (52.8\%), 0-5\% in $103 / 305(33.8 \%)$ and $5-30 \%$ in $41 / 305(13.4 \%)$ (Figure $3 A-3 C$ ). The median age of donors with steatosis was $31.6(28.9,37.4)$ years old, male accounted for $58.5 \%$ and BMI $24.2(20.9,25.4) \mathrm{kg} / \mathrm{m}^{2}$. Median ALT, aspartate aminotransferase and gamma-glutamyl transpeptidase of these 41 donors with steatosis was 20.0 (16.0,27.0), $19.6(17.1,23.8)$ and $18.0(15.0,25.0)$ IU/L, respectively. $36 / 41(87.8 \%)$ ALT was less than 40 IU/L. It is worthy to point out that age distribution of 41 donors with steatosis was: $18 / 41$ (43.9\%) in $20-30$ years old, $20 / 41$ (48.8\%) in $30-40$ years old, and $3 / 41(7.3 \%)$ in $40-50$ years old. Distribution of BMI of these steatotic donors was $<23 \mathrm{~kg} / \mathrm{m}^{2}: 14$ (34.1\%), $23-25 \mathrm{~kg} / \mathrm{m}^{2}: 15$ (36.6\%) and $>25 \mathrm{~kg} / \mathrm{m}^{2}: 12(29.3 \%)$.

IPI was present in $85 / 305$ (27.9\%): mild in $80 / 305$ (26.2\%) and mild to moderate in 5/305 (1.7\%). 220/305 $(72.1 \%)$ had no portal inflammation (Figure $3 D-3 F)$. Fourteen donors (4.6\%) had both steatosis $(\geq 5 \%)$ and IPI $(\geq$ grade 1) in their livers. No obvious ballooning hepatocytes were perceived.

\section{Impact of hepatic steatosis and/or IPI on the evolution of recipients' ALT and TBIL at 1 week, 2 weeks and 4 weeks post LDLT}

As compared to non-steatosis group, steatosis group had a comparable serum ALT level at 1 week, 2 weeks and 


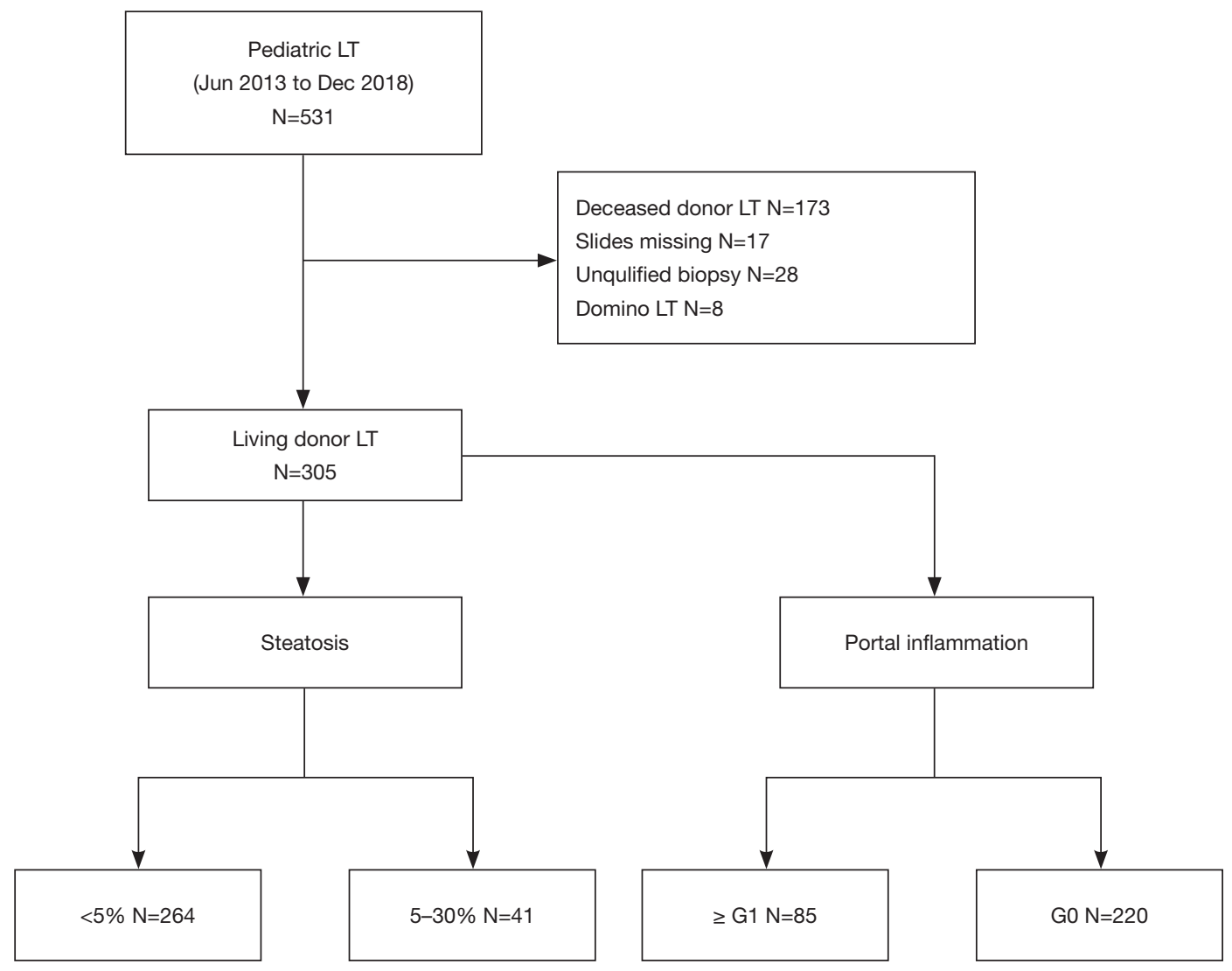

Figure 1 Flowchart of the study. A total of 305 out of 358 living donor pediatric liver transplantation were enrolled in this study, 41 out of 305 cases received living donor livers with 5-30\% steatosis, 85 out of 305 cases received living donor livers with mild or mild to moderate idiopathic portal inflammation $(\mathrm{G} \geq 1)$. LT, liver transplantation.

4 weeks post LDLT (all P value $>0.05$ ). Additionally, steatosis group had trend higher level of serum TBIL at 1 week, 2 weeks and 4 weeks post LDLT in comparison with non-steatosis group, however it was not statistically significant $(\mathrm{P}=0.247$ for $\mathrm{ALT}$ and $\mathrm{P}=0.733$ for TBIL; Table 2).

As compared to non-IPI group, the IPI group had a trend lower serum ALT and TBIL level at 1 week, 2 weeks and 4 weeks post LDLT without statistic difference $(\mathrm{P}=0.776$ for ALT and $\mathrm{P}=0.724$ for TBIL; Table 2).

As compared to recipients who received living donor livers without steatosis and IPI (non-steatosis and non-IPI group), those received living donor livers with both steatosis and IPI (steatosis and IPI group) had no significant difference of serum ALT and TBIL level at 1 week, 2 weeks and 4 weeks post LDLT ( $\mathrm{P}=0.428$ for ALT and $\mathrm{P}=0.640$ for TBIL; Table 3).

\section{Impact of hepatic steatosis and/or IPI on short- and long- term complications post LDLT}

Steatosis group had trend reduced overall short-term complications $(<1-$ month post-LDLT) as compared to non-steatosis group $(25.0 \%$ vs. $37.3 \% \mathrm{P}=0.131)$ without significant difference. When further dividing overall complications into surgical and acute rejection/ infection associated subgroups, steatosis group had a trend lower proportion of acute rejection/infection related complications $(22.0 \%$ vs. $32.2 \%, \mathrm{P}=0.186)$ but trend higher surgical complications ( $14.6 \%$ vs. $11.7 \%, \mathrm{P}=0.598)$ as compared to non-steatosis group without significant difference. Steatosis had trend higher overall long-term complications as compared to non-steatosis groups without significance $(\mathrm{P}=0.856$; Table 2$)$. 

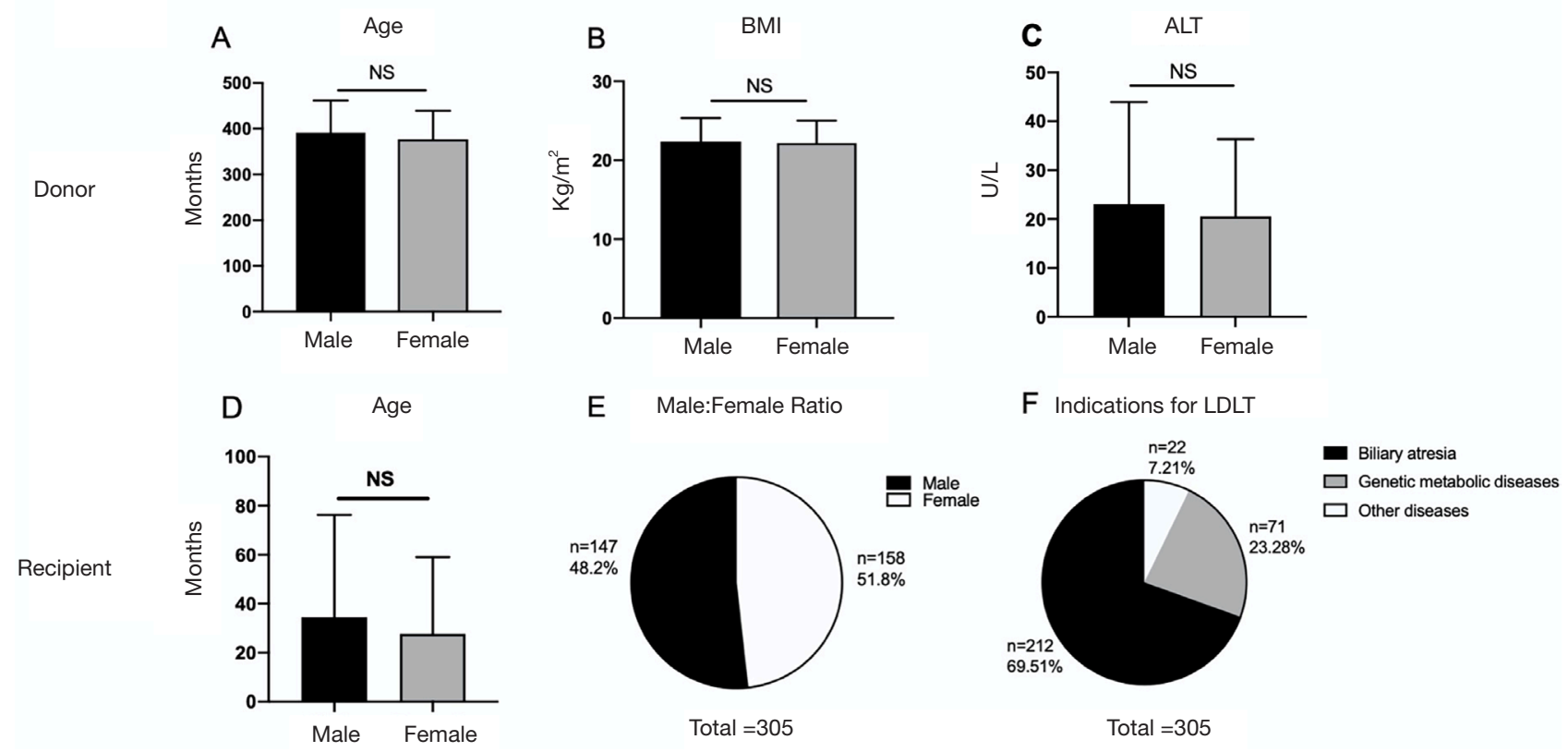

Figure 2 Baseline and demographic data of donor and recipients. Age of living donors was 31.2 (28.0-35.8) years old without significant difference between male and female donors (A). Medium BMI was $22.2(20.1-24.4) \mathrm{kg} / \mathrm{m}^{2}$ (B) and average serum ALT (C) was within normal range. Age of recipients was 1.0 (0.6-3.6) years old (D) and male patient accounted for $51.8 \%$ (E). The main indication for liver transplantation was biliary atresia (F). BMI, body mass Index; ALT, alanine aminotransferase.

IPI group had marginal higher proportion of overall short-term complications ( $44.1 \%$ vs. $32.4 \%, \mathrm{P}=0.059$ ), including both infection/rejection $(38.8 \%$ vs. $27.7 \%$, $\mathrm{P}=0.060)$ and surgical complications $(15.3 \%$ vs. $10.9 \%$, $\mathrm{P}=0.293$ ) in comparison to non-IPI group (no statistical difference). Similar trend was observed in long-term complications between IPI and non-IPI groups without significant difference statistically (Table 2).

Steatosis and IPI group didn't have significantly higher incidence of overall short- and long-term complications than non-steatosis and non-IPI group (Table 3).

\section{Impact of hepatic steatosis and/or IPI on graft/overall survival post LDLT}

The graft survival of this cohort was $96.7 \%, 95.2 \%$ and $95.2 \%$ and the overall survival of this cohort was $97.1 \%$, $96.3 \%$ and $96.3 \%$ respectively at 1-, 3-, 5-year post LDLT. Steatosis group $(97.6 \%, 94.6 \%$ and $94.6 \%)$ and nonsteatosis group (96.6\%, 95.3\% and $95.3 \%$ ) had comparable graft survival $(\mathrm{P}=0.935)$ and overall survival 1-, 3-, 5-year post LDLT (steatosis group 97.6\%, 97.6\% and $97.6 \%$ vs. non steatosis group $97.0 \%, 96.1 \%$ and $96.1 \%, \mathrm{P}=0.674$
(Table 2, Figure 4A).

Both IPI and non-IPI group had similar 1-, 3-, 5-year graft survival $(94.1 \%, 94.1 \%$ and $94.1 \%$ vs. $97.7 \%, 95.6 \%$, 95.6\%, $\mathrm{P}=0.456)$ and $1,3,5$-year overall survival $(95.3 \%$, $95.3 \%$ and $95.3 \%$ vs. $97.7 \%, 96.8 \%$ and $96.8 \%, \mathrm{P}=0.506$ ) post LDLT, despite the IPI group appeared to be slightly lower than the non-IPI group (Table 2, Figure 4B).

Recipients received living donor livers with both IPI and steatosis had no synergetic negative effects on 1-, 3-, 5-year graft survival $(100.0 \%, 100.0 \%$ and $100.0 \%$ vs. $97.9 \%$, $96.2 \%, 96.2 \%, \mathrm{P}=0.447)$ and $1-, 3-, 5$-year overall survival $(100.0 \%, 100.0 \%$ and $100.0 \%$ vs. $97.9 \%, 96.8 \%$ and $96.8 \%$, $\mathrm{P}=0.671)$ post LDLT compared with non-steatosis and nonIPI group (Table 3, Figure 4C).

\section{Impact steatosis and/or IPI on donor-recipient matching stratified by PELD score}

Using PELD score medium value 16 in the cohort as the cut off score, we divided recipients into higher PELD score group (PELD $\geq 16$ ) and lower PELD score group (PELD $<16)$. Our results showed higher PELD score recipients had trend lower graft survival $(94.8 \%, 93.1 \%$ and $93.1 \%$ vs. 
Table 1 Baseline and demographic characteristics of donors and recipients

\begin{tabular}{lcc}
\hline & Donor $(\mathrm{N}=305)$, median $(\mathrm{Q} 1-\mathrm{Q} 3) /(\%)$ & Recipient $(\mathrm{N}=305)$, median $(\mathrm{Q} 1-\mathrm{Q} 3) /(\%)$ \\
\hline Male $(\%)$ & 42.3 & 51.8 \\
Age (Months) & $374.0(336.0-429.0)$ & $12.0(7.0-43.0)$ \\
BMI $\left(\mathrm{Kg} / \mathrm{m}^{2}\right)$ & $22.2(20.1-24.4)$ & $16.1(14.7-17.5)$ \\
ALT $(\mathrm{U} / \mathrm{L})$ & $16.0(12.0-24.0)$ & $98.0(44.0-190.0)$ \\
AST $(\mathrm{U} / \mathrm{L})$ & $19.6(16.1-23.7)$ & $\mathrm{NA}$ \\
GGT $(\mathrm{U} / \mathrm{L})$ & $17.0(12.0-24.0)$ & $\mathrm{NA}$ \\
TBIL $(\mu \mathrm{mol} / \mathrm{L})$ & $\mathrm{NA}$ & $164.9(18.5-377.7)$ \\
PELD & - & $16.0(5.0-26.0)$
\end{tabular}

BMI, body mass Index; ALT, alanine aminotransferase; AST, aspartate aminotransferase; GGT, gamma-glutamyl transpeptidase; TBIL, total bilirubin; PELD, pediatric end-stage liver disease.
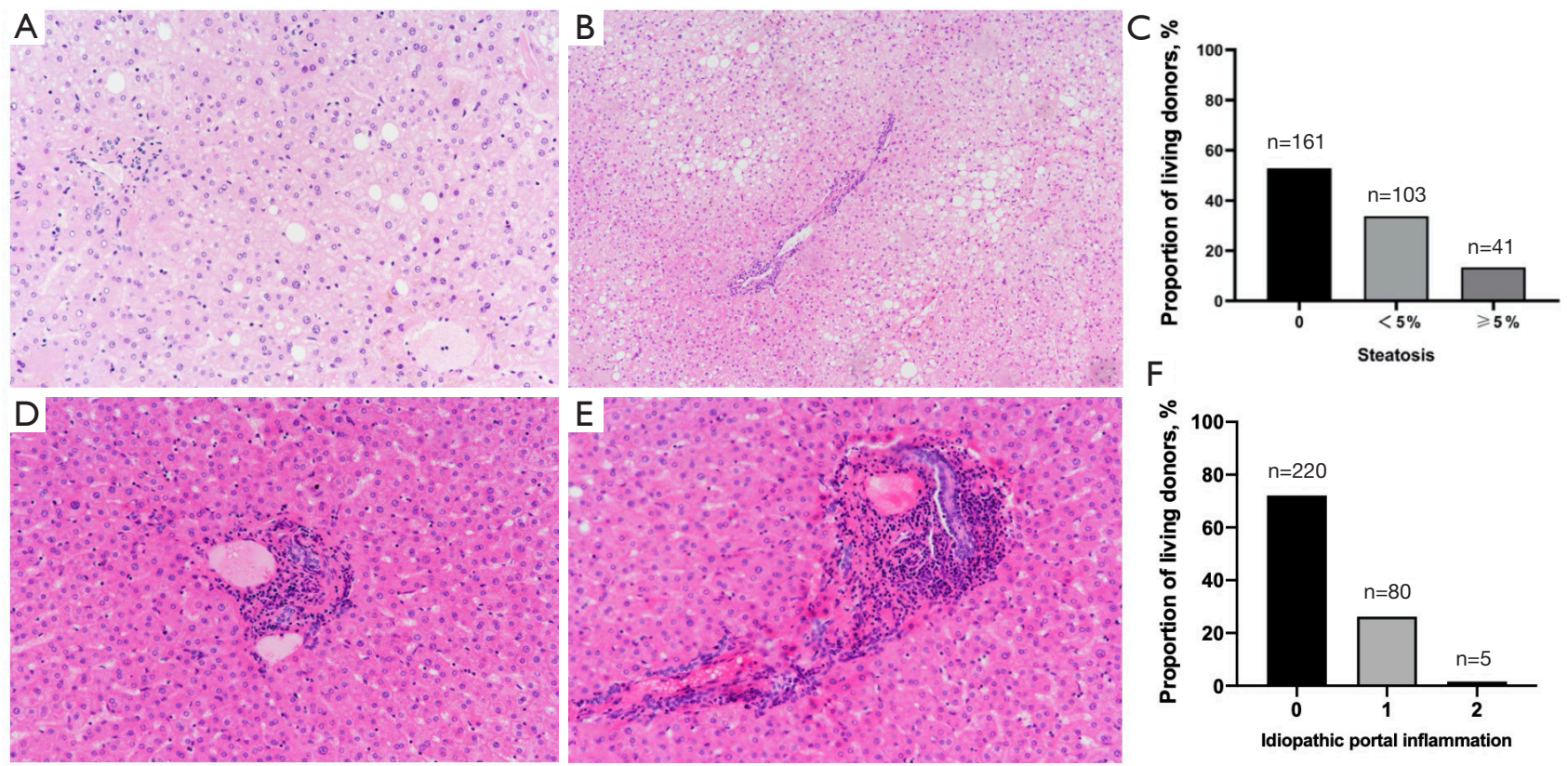

Figure 3 Histological analysis of 305 living donor wedged liver biopsy. One hundred and sixty-one out of 305 living donor liver had no steatosis, 103 cases had steatosis less than $5 \%$ (A) and 41 cases had steatosis from $5 \%$ to $30 \%$ (B,C). As for portal inflammation, 220 donor liver had no portal inflammation, 80 donor had mild portal inflammation (D) and 5 cases had mild to moderate portal inflammation (E,F). HE staining (100x).

98.0\%, 97.2\% and $97.2 \%, \mathrm{P}=0.115$, Figure $5 A$ ) and overall survival rate $(95.5 \%, 94.6 \%$ and $94.6 \%$ vs. $98.0 \%, 98.0 \%$ and $98.0 \%, \mathrm{P}=0.146$, Figure 5B) compared with low PELD score recipients $(\mathrm{PELD}<16)$ without statistic difference. When donor livers with steatosis (no IPI) were distributed to the higher PELD score recipients $(\geq 16)$, these patients had similar graft survival $(100.0 \%, 100.0 \%$ and $100.0 \%$ vs. $94.7 \%, 88.4 \%$ and $88.4 \%, \mathrm{P}=0.102$, Figure $5 C$ ) and overall survival $(100.0 \%, 100.0 \%$ and $100.0 \%$ vs. $94.7 \%$, $94.7 \%$ and $94.7 \%, \mathrm{P}=0.255$, Figure 5D) in comparison to lower PELD score recipients. When donor livers with IPI (no steatosis) were distributed to the higher PELD 


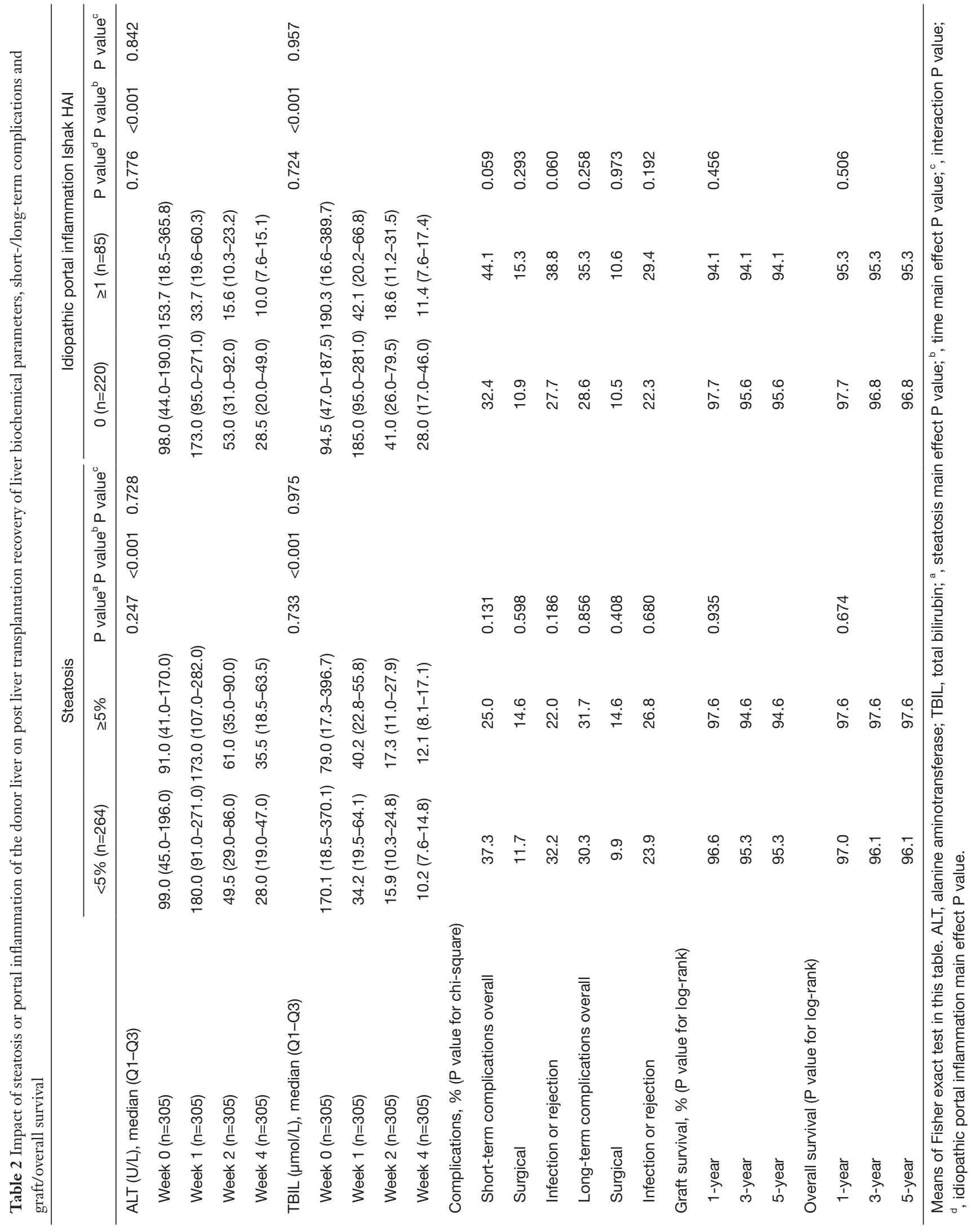




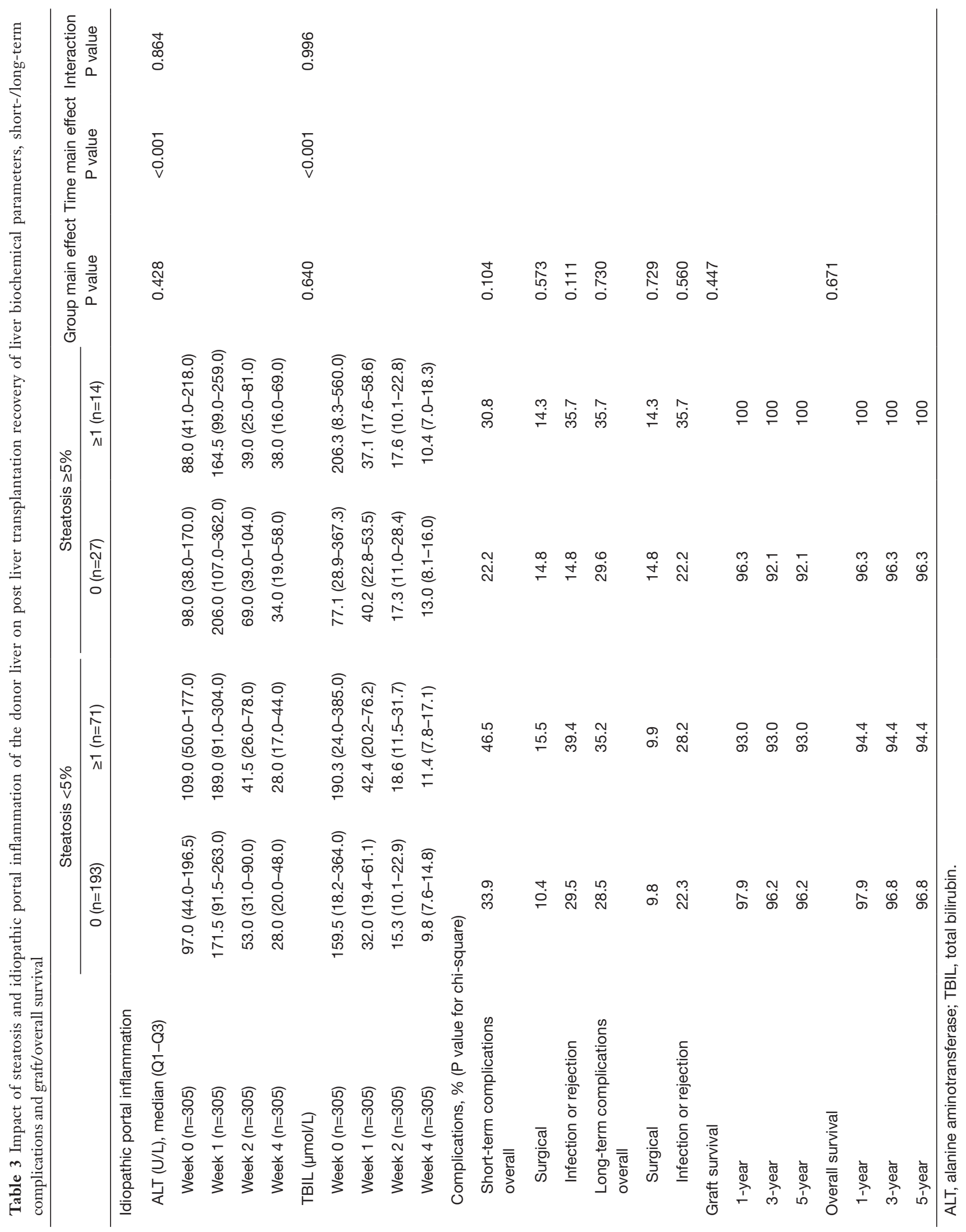




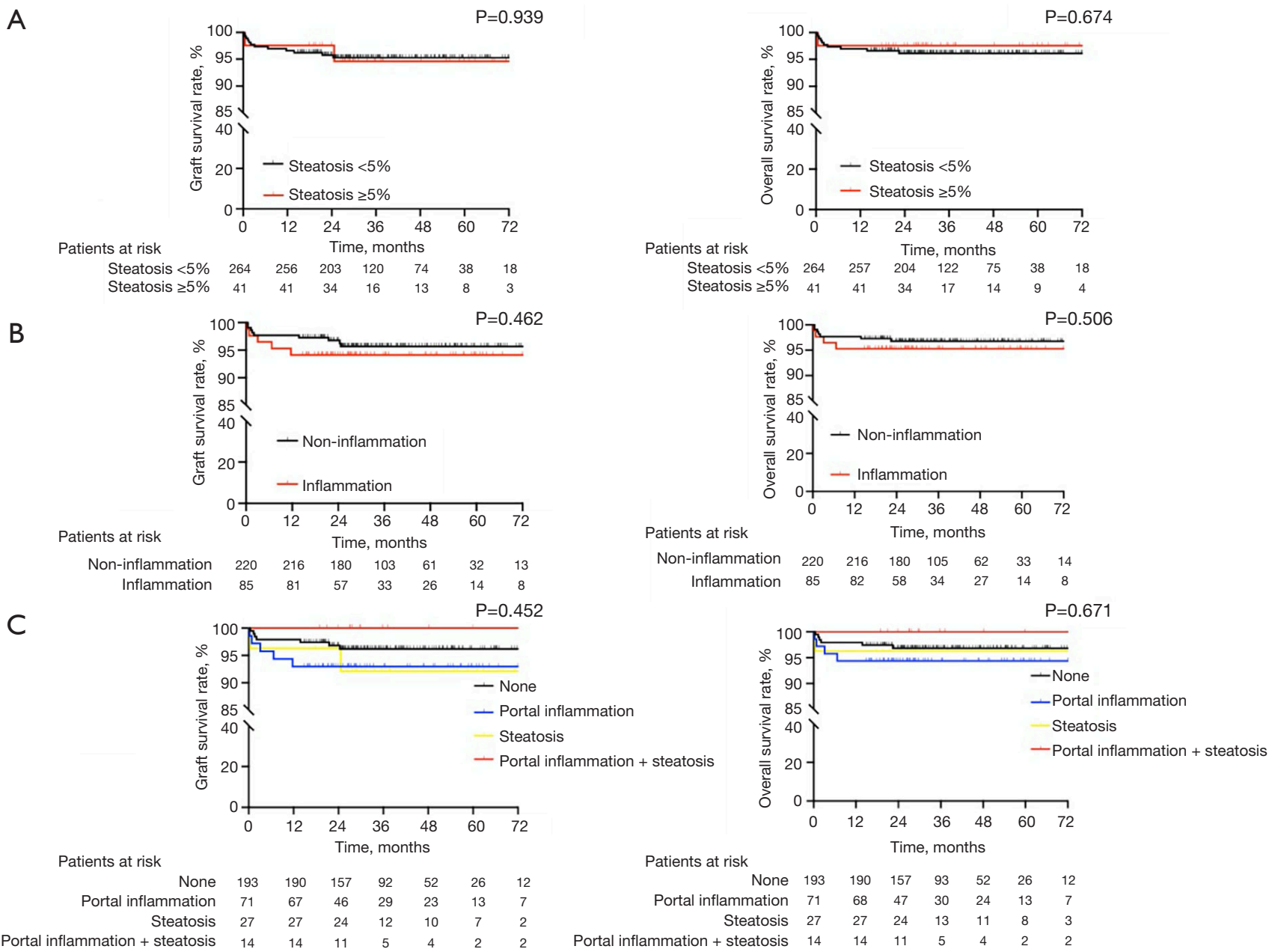

Figure 4 Comparative graft survival/overall survival between recipients receiving living donor liver with/without steatosis and/or idiopathic portal inflammation. Recipients received living donor liver with steatosis had no negative effects on graft survival and overall survival, without significant difference (A). Recipients had living donor liver with idiopathic portal inflammation had slightly lower graft or overall survival (B). Unexpectedly, recipients who had living donor liver with both steatosis and portal inflammation didn't have syngenetic negative effects on recipients' graft and overall survival (C).

score recipients $(\geq 16)$, these patients had trend lower graft survival $(90.5 \%, 90.5 \%$ and $90.5 \%$ vs. $97.7 \%, 97.7 \%$ and $97.7 \%, \mathrm{P}=0.174$, Figure $5 E)$ and overall survival $(92.9 \%$, $92.9 \%$ and $92,9 \%$ vs. $97.7 \%, 97.7 \%$ and $97.7 \%, \mathrm{P}=0.311$, Figure $5 F$ ) in comparison to lower PELD score recipients.

\section{Identification of other clinical independent risk factor (s) for graft/overall survival in pediatric LDLT}

Cox regression analysis revealed that only short-term complications $(\mathrm{HR}=3.343,95 \% \mathrm{CI}, 1.12-9.977, \mathrm{P}=0.031$ ) were independently associated with graft survival (Table 4). No additional independent risk factors associated with overall survival were identified. The subsequent logistic regression analysis showed that younger age $(\mathrm{HR}=0.990$, 95\% CI, 0.982-0.977, $\mathrm{P}=0.007$ ), biliary atresia (HR $=1.874,95 \%$ CI, $1.091-3.218, \mathrm{P}=0.023)$ and higher PELD score $(\mathrm{HR}=1.732,95 \% \mathrm{CI}, 1.076-2.789, \mathrm{P}=0.024)$ were independently associated with short-term complications. Multi-variate regression analysis revealed that only high PELD score was independently associated with short-term complication (1.742, 95\% CI, 1.064-2.900, $\mathrm{P}=0.033$; Table 5). 

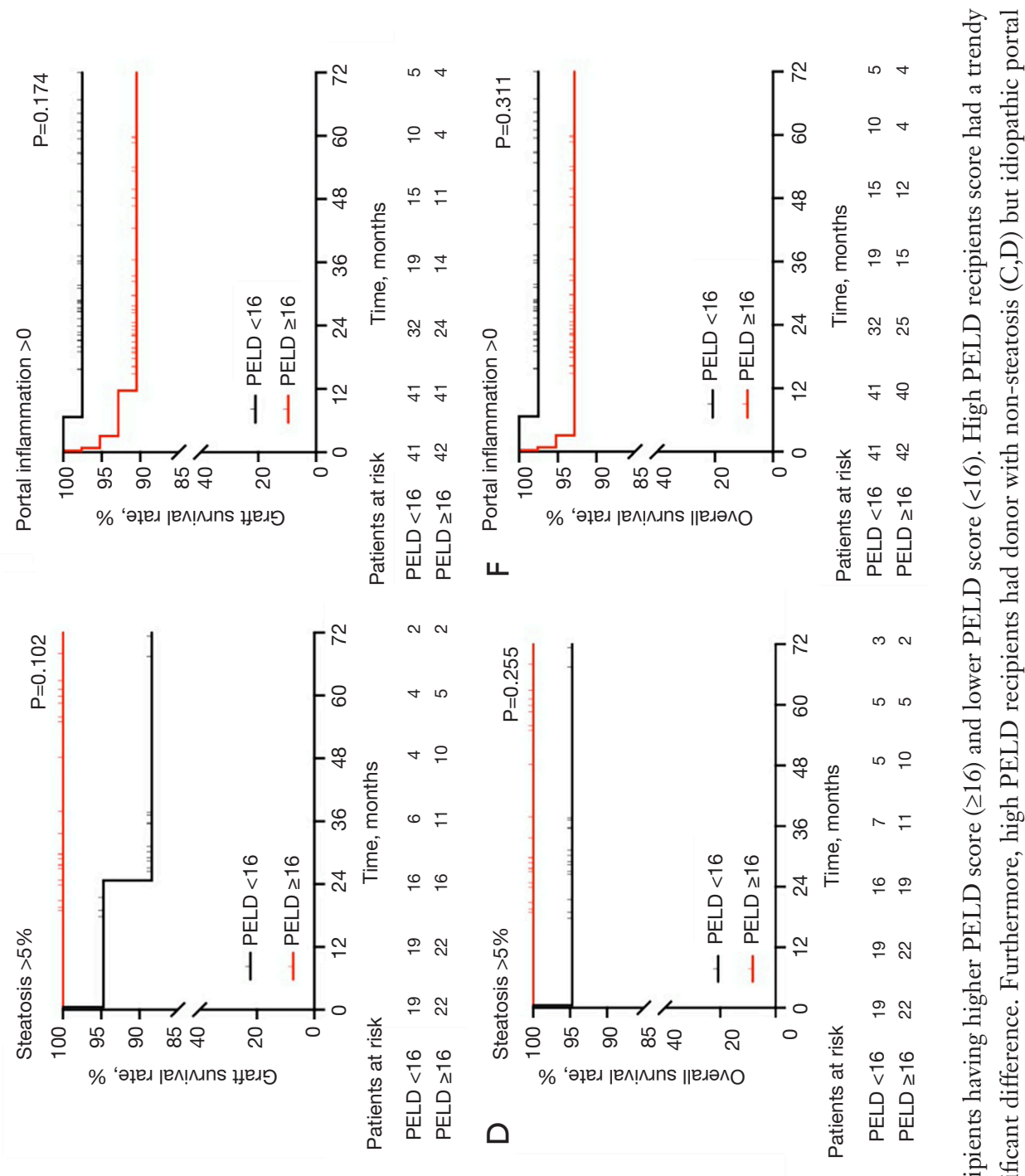

$\cup$
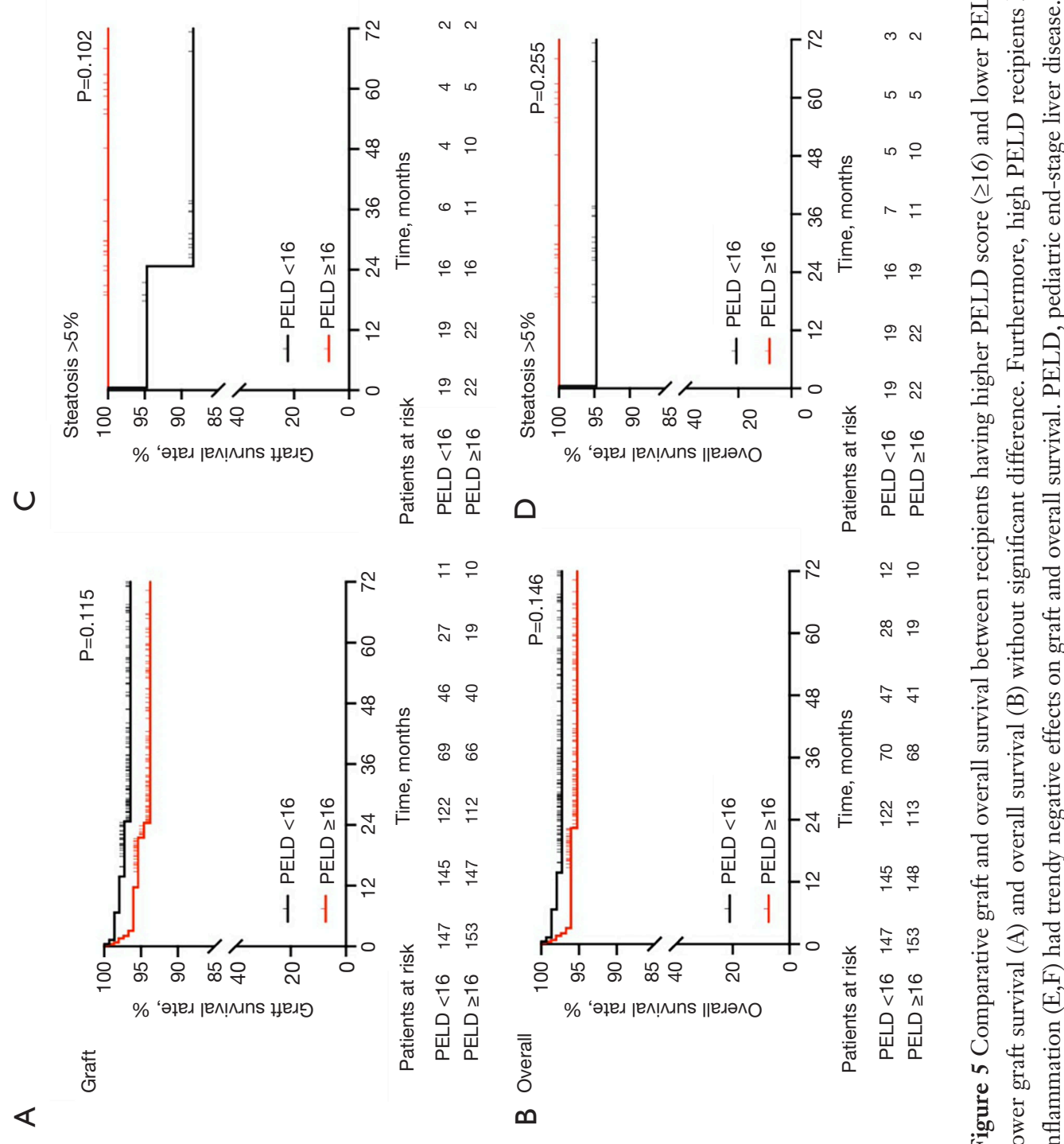
Table 4 Clinical and histopathological risk factors independently associated with graft survival post living donor pediatric liver transplantation

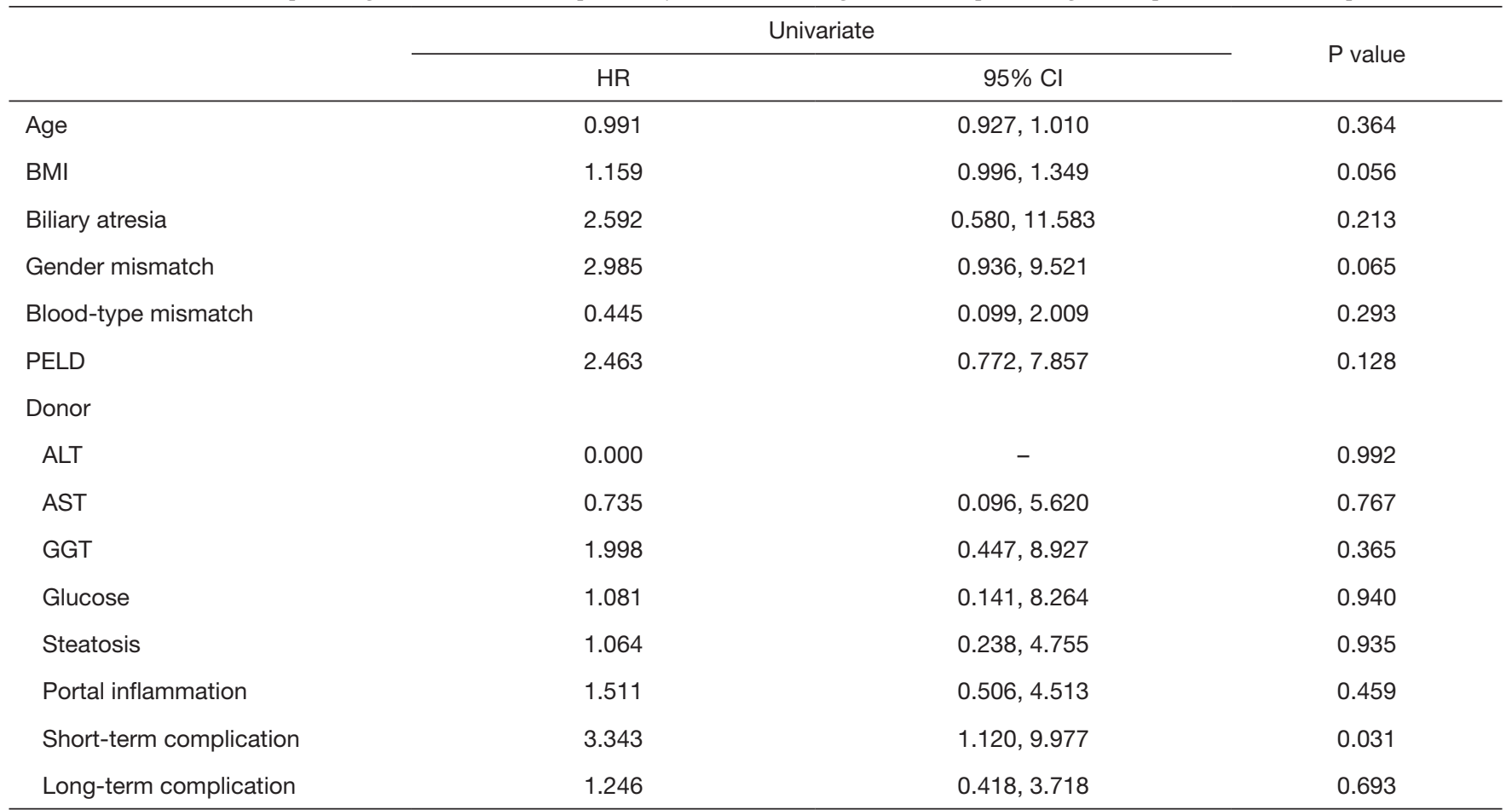

BMI, body mass Index; PELD, pediatric end-stage liver disease; ALT, alanine aminotransferase; AST, aspartate aminotransferase; GGT, gamma-glutamyl transpeptidase.

Table 5 Clinical and histological risk factors independently associated with short-term complications post living donor pediatric liver transplantation

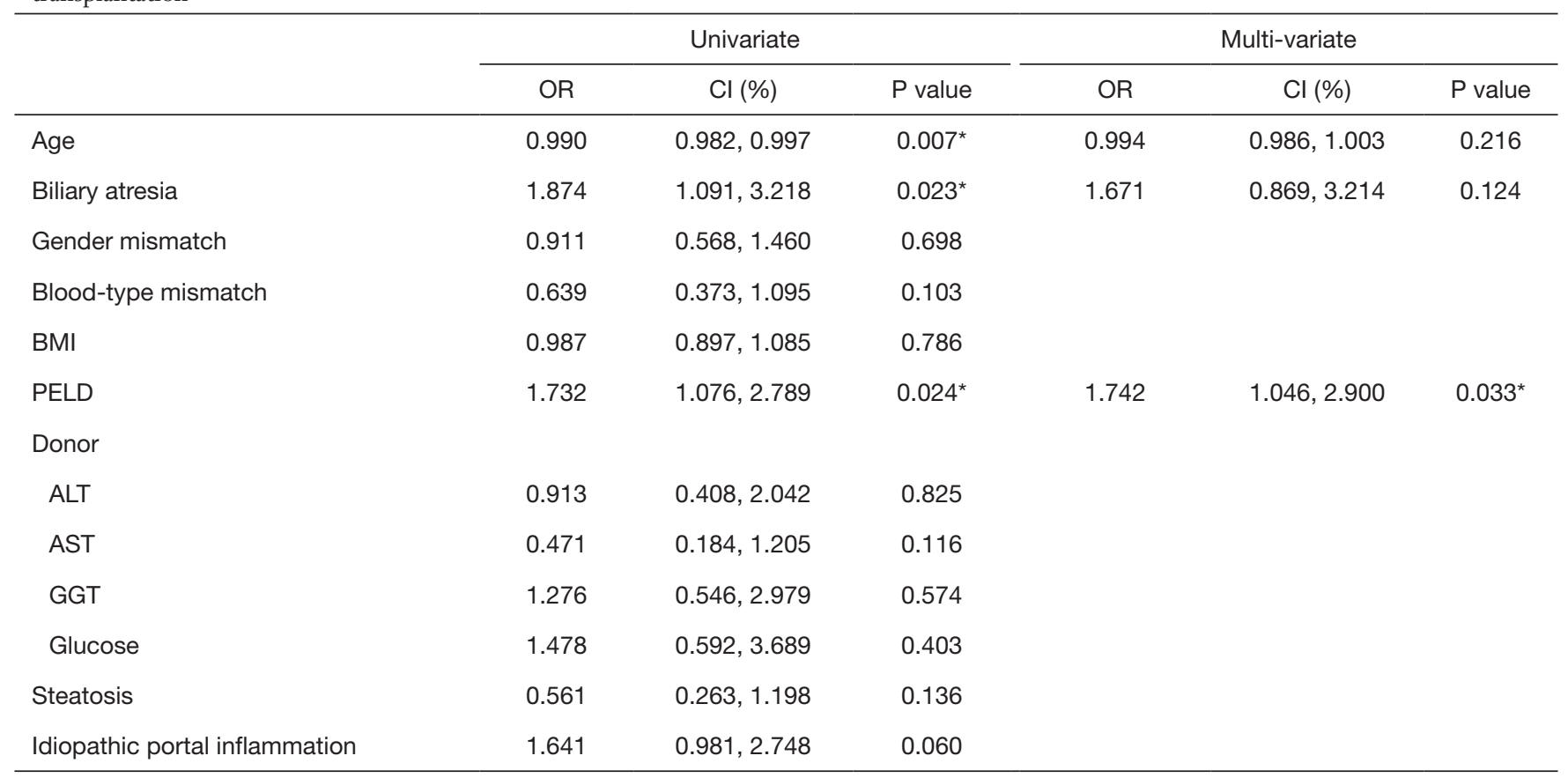

${ }^{*}$, statistically significant. BMI, body mass Index; PELD, pediatric end-stage liver disease; ALT, alanine aminotransferase; AST, aspartate aminotransferase; GGT, gamma-glutamyl transpeptidase. 


\section{Discussion}

In our study, 41 out of 305 donors (13.4\%) had NAFLD according to the histological criterion, i.e., steatosis $\geq 5 \%$. A majority of subjects had low BMI $\left(\leq 25 \mathrm{~kg} / \mathrm{m}^{2}\right)$ and did not have other risk factors, including alcohol overconsumption (24). Since there were no lobular inflammation, ballooning hepatocytes nor fibrosis histologically, these donors should be diagnosed of having 'lean' NAFLD, specifically nonalcoholic fatty liver (NAFL) (25).

NAFLD is a well-recognized liver disorder and the most common cause of chronic liver disease. It is composed of NAFL and nonalcoholic steatohepatitis. NAFL can be diagnosed either by imaging or histopathology, while the diagnosis of nonalcoholic steatohepatitis requires histopathology, the golden standard (26). The estimated prevalence of NAFLD varies from $25.2 \%$ globally to up to $20.0 \%$ in China in $2020(24,27)$. NAFLD is often linked with metabolic syndromes, obesity and being overweight, but can also occur in normal weight population. 'Lean' NAFLD was initially labelled as a disorder predominantly involving Asian population. However, it can also occur in other ethnicities. The prevalence of 'lean' NAFLD in general population varies from $10.0 \%$ to $20.0 \%$. Most of these large-scaled studies were image-based, i.e., $7.0 \%$ in the USA (24), 7.3\% in China (28), 16.1\% in Korea (29), 15.2\% in Japan (30) and $19.3 \%$ in Hong Kong (31). Comparing to these image-based NAFLD studies, our study is the largest histopathology-approved NAFLD cohort among healthy Chinese selected for living liver donation. These subjects had nearly normal liver biochemistries and went through restrict selective criteria for living organ donation. The data here reasonably reflect current NAFLD, particularly NAFL status in healthy donor population in China.

For overweight or obesity donors, we recommend that a balanced nutrition, moderate-intensity physical activity and $5-10 \%$ weight loss before LT in order to reduce the extent of fatty liver disease (32). The donors for dietary changes recommend reducing the intake of saturated fat to $<7 \%$ of total calories, reducing trans-fat intake, and maintaining dietary cholesterol intake at $<200 \mathrm{mg} /$ day and total fat at $25 \%$ to $35 \%$ of total calories. Moreover, engaging in regular moderate-intensity physical activity for at least 30 minutes for more than 5 days/week is recommended for these donors too (33).
Furthermore, our study showed that steatosis below 30\% from living donors did not significantly impact on graft and overall survival. This result compares favorably with previous adult whole liver LT studies, where steatosis less than $30 \%$ were as generally accepted $(18,19,34)$. Our results add strong evidence to the fact that mild steatosis does not significantly affect clinical outcomes in pediatric LDLT $(13,35)$.

Idiopathic mild portal inflammation characterized by predominantly mononuclear inflammatory cells infiltration in portal tracts was frequently observed and its prevalence was up to $16 \%$ in 'healthy living donors' (15). Clinical significance of this mild IPI during donor liver assessment and its effects on recipients' clinical outcomes have not been well studied yet. In the present study, our data for the first time showed that mild IPI alone didn't have significant negative effects on post LDLT liver biochemical parameters normalization. However, the IPI group did have trend higher incidence of short- and long-term complications and lower graft and overall survival rates compared to non-IPI group, though without statistical significance. The surgical complications were divided into biliary complications and vascular complications. Biliary complications (bile leakage, cholangitis and biliary anastomotic stenosis, etc.) accounted for $7.2 \%(22 / 305)$, vascular complications (portal vein embolism, hepatic artery embolism, etc.) accounted for $5.2 \%(16 / 305)$. Additionally, in our study, about $4.6 \%$ donor livers had both steatosis (5-30\%) and IPI $(\geq 1)$. However, the combined histological features had no syngenetic disadvantage effect on recipients' graft and overall survival rate.

Organ-recipient matching allocation is another ongoing topic in LT. When we further stratified recipients into two subgroups according to PELD score and found that higher PELD score group (PELD $>16$ ) received living donor livers with IPI but not steatosis had negative impact on graft/ overall survival, though there was no different statistically. The results were in parallel to the previous study that high quality donor liver should be allocated to sicker recipients, which may benefit these patients in terms of reducing post LDLT complications and graft loss (36).

This is the largest cohort systemically investigating hepatic steatosis and IPI in healthy living donors, and their impact on clinical outcomes. However, there are limitations in our study. Firstly, since the majority of donors were 20 to 
30 years old, and only donors with $\leq 30 \%$ hepatic steatosis were selected for donation, the findings on NAFLD prevalence can only reflect this particular population. Secondly, it was a single center retrospective study, the results need to be further validated by other centers or ethnic population.

\section{Conclusions}

This study validates that mild steatosis does not affect recipient's graft and overall survival. However, mild IPI does have trend negative impact on graft and overall survival, especially when theses organs were allocated to higher PELD score recipients. Steatosis and IPI do not have syngenetic effects on graft and overall survival. This study aids in better care in pediatric living donor LT.

\section{Acknowledgments}

We are deeply grateful to Prof. em.Jan LERUT from Institute de Recherche Clinique (IREC), Université Catholique Louvain (UCL) Brussels, Belgium for his generous help in critical revision and language polishing. Funding: This work was funded by the Digestive Medical Coordinated Development Center of Beijing Hospitals Authority (No. XXZ0301).

\section{Footnote}

Reporting Checklist: The authors have completed the STROBE reporting checklist. Available at https://hbsn. amegroups.com/article/view/10.21037/hbsn-20-685/rc

Data Sharing Statement: Available at https://hbsn. amegroups.com/article/view/10.21037/hbsn-20-685/dss

Conflicts of Interest: All authors have completed the ICMJE uniform disclosure form (available at https://hbsn. amegroups.com/article/view/10.21037/hbsn-20-685/ coif). ZZ serves as an unpaid editorial board member of Hepatobiliary Surgery and Nutrition. The other authors have no conflicts of interest to declare.

Ethical Statement: The authors are accountable for all aspects of the work in ensuring that questions related to the accuracy or integrity of any part of the work are appropriately investigated and resolved. The study was conducted in accordance with the Declaration of Helsinki (as revised in 2013). The study was approved by institutional ethics board of Beijing Friendship Hospital (No. 2018-P2207-01) and informed consent was taken from all individual participants.

Open Access Statement: This is an Open Access article distributed in accordance with the Creative Commons Attribution-NonCommercial-NoDerivs 4.0 International License (CC BY-NC-ND 4.0), which permits the noncommercial replication and distribution of the article with the strict proviso that no changes or edits are made and the original work is properly cited (including links to both the formal publication through the relevant DOI and the license). See: https://creativecommons.org/licenses/by-nc-nd/4.0/.

\section{References}

1. Xiao J, Wang F, Wong NK, et al. Global liver disease burdens and research trends: Analysis from a Chinese perspective. J Hepatol 2019;71:212-21.

2. Estes C, Anstee QM, Arias-Loste MT, et al. Modeling NAFLD disease burden in China, France, Germany, Italy, Japan, Spain, United Kingdom, and United States for the period 2016-2030. J Hepatol 2018;69:896-904.

3. Estes C, Razavi H, Loomba R, et al. Modeling the epidemic of nonalcoholic fatty liver disease demonstrates an exponential increase in burden of disease. Hepatology 2018;67:123-33.

4. Firl DJ, Sasaki K, McVey J, et al. Improved Survival following Living Donor Liver Transplantation for Pediatric Acute Liver Failure: Analysis of the US National Registry over Two Decades. Liver Transpl 2019;25:1241-50.

5. Montenovo MI, Bambha K, Reyes J, et al. Living liver donation improves patient and graft survival in the pediatric population. Pediatr Transplant 2019;23:e13318.

6. Kehar M, Parekh RS, Stunguris J, et al. Superior Outcomes and Reduced Wait Times in Pediatric Recipients of Living Donor Liver Transplantation. Transplant Direct 2019;5:e430.

7. Zhang R, Zhu ZJ, Sun LY, et al. Outcomes of Pediatric Liver Transplantation: Deceased Donor Liver Transplantation vs Living Donor Liver Transplantation. Transplant Proc 2018;50:3601-5.

8. Angele MK, Rentsch M, Hartl WH, et al. Effect of graft steatosis on liver function and organ survival after liver 
transplantation. Am J Surg 2008;195:214-20.

9. Briceño J, Ciria R, Pleguezuelo $M$, et al. Impact of donor graft steatosis on overall outcome and viral recurrence after liver transplantation for hepatitis $\mathrm{C}$ virus cirrhosis. Liver Transpl 2009;15:37-48.

10. Jun MJ, Shim JH, Kim SY, et al. Clinical implications of preoperative and intraoperative liver biopsies for evaluating donor steatosis in living related liver transplantation. Liver Transpl 2014;20:437-45.

11. Spitzer AL, Lao OB, Dick AA, et al. The biopsied donor liver: incorporating macrosteatosis into high-risk donor assessment. Liver Transpl 2010;16:874-84.

12. Angelico M. Donor liver steatosis and graft selection for liver transplantation: a short review. Eur Rev Med Pharmacol Sci 2005;9:295-7.

13. Li HY, Wei YG, Li B, et al. Impact of graft steatosis on the post-transplantation biliary complications for living donor liver transplant recipients in China. Hepatogastroenterology 2012;59:1194-7.

14. Liu Z, Jia J, Ning H, et al. Systematic Evaluation of the Safety Threshold for Allograft Macrovesicular Steatosis in Cadaveric Liver Transplantation. Front Physiol 2019;10:429.

15. Pungpapong S, Krishna M, Abraham SC, et al. Clinicopathologic findings and outcomes of liver transplantation using grafts from donors with unrecognized and unusual diseases. Liver Transpl 2006;12:310-315.

16. Ryan CK, Johnson LA, Germin BI, et al. One hundred consecutive hepatic biopsies in the workup of living donors for right lobe liver transplantation. Liver Transpl 2002;8:1114-22.

17. Ayvazoglu Soy EH, Boyvat F, Ozdemir BH, et al. Liver Biopsy Results in Potential Donor Evaluation in Living Related Liver Transplant. Exp Clin Transplant 2018;16 Suppl 1:35-37.

18. Cuomo O, Perrella A, Pisaniello D, et al. Evidence of liver histological alterations in apparently healthy individuals evaluated for living donor liver transplantation. Transplant Proc 2008;40:1823-6.

19. Tran TT, Changsri C, Shackleton CR, et al. Living donor liver transplantation: histological abnormalities found on liver biopsies of apparently healthy potential donors. J Gastroenterol Hepatol 2006;21:381-3.

20. Jackson KR, Motter JD, Haugen CE, et al. Minimizing Risks of Liver Transplantation with Steatotic Donor Livers by Preferred Recipient Matching. Transplantation 2020;104:1604-11.

21. Kleiner DE, Brunt EM, Van Natta M, et al. Design and validation of a histological scoring system for nonalcoholic fatty liver disease. Hepatology 2005;41:1313-21.

22. Ishak K, Baptista A, Bianchi L, et al. Histological grading and staging of chronic hepatitis. J Hepatol. 1995;22:696-9.

23. Jiang YZ, Sun LY, Zhu ZJ, et al. Perioperative characteristics and management of liver transplantation for isolated methylmalonic acidemia-the largest experience in China. Hepatobiliary Surg Nutr 2019;8:470-9.

24. Younossi ZM, Stepanova M, Negro F, et al. Nonalcoholic fatty liver disease in lean individuals in the United States. Medicine (Baltimore) 2012;91:319-27.

25. Fan JG, Wei L, Zhuang H, National Workshop on Fatty L, Alcoholic Liver Disease CSoHCMA, Fatty Liver Disease Expert Committee CMDA. Guidelines of prevention and treatment of nonalcoholic fatty liver disease (2018, China). J Dig Dis 2019;20:163-73.

26. Bedossa P, Consortium FP. Utility and appropriateness of the fatty liver inhibition of progression (FLIP) algorithm and steatosis, activity, and fibrosis (SAF) score in the evaluation of biopsies of nonalcoholic fatty liver disease. Hepatology 2014;60:565-75.

27. Zhu JZ, Zhou QY, Wang YM, et al. Prevalence of fatty liver disease and the economy in China: A systematic review. World J Gastroenterol 2015;21:5695-706.

28. Xu C, Yu C, Ma H, et al. Prevalence and risk factors for the development of nonalcoholic fatty liver disease in a nonobese Chinese population: the Zhejiang Zhenhai Study. Am J Gastroenterol 2013;108:1299-304.

29. Kim NH, Kim JH, Kim YJ, et al. Clinical and metabolic factors associated with development and regression of nonalcoholic fatty liver disease in nonobese subjects. Liver Int 2014;34:604-611.

30. Nishioji K, Sumida Y, Kamaguchi M, et al. Prevalence of and risk factors for non-alcoholic fatty liver disease in a non-obese Japanese population, 2011-2012. J Gastroenterol 2015;50:95-108.

31. Wei JL, Leung JC, Loong TC, et al. Prevalence and Severity of Nonalcoholic Fatty Liver Disease in NonObese Patients: A Population Study Using ProtonMagnetic Resonance Spectroscopy. Am J Gastroenterol 2015;110:1306-1314; quiz 1315.

32. Anania C, Perla FM, Olivero F, et al. Mediterranean diet and nonalcoholic fatty liver disease. World J Gastroenterol 2018;24:2083-2094.

33. Kwak MS, Kim D. Non-alcoholic fatty liver disease and lifestyle modifications, focusing on physical activity. Korean J Intern Med 2018;33:64-74.

34. Perez-Daga JA, Santoyo J, Suarez MA, et al. Influence 
of degree of hepatic steatosis on graft function and postoperative complications of liver transplantation.

Transplant Proc 2006;38:2468-70.

35. Sood V, Alam S. Outcome of Pediatric Living Donor Liver Transplantation in India. Indian Pediatr 2018;55:349-50.

Cite this article as: Zhao X, He Y, Liu J, Zhang Q, Liu L, Qu W, Liu Y, Zeng Z, Zhang H, Jia J, Sun L, Wei L, Zhu Z. Impact of living donor liver with steatosis and idiopathic portal inflammation on clinical outcomes in pediatric liver transplantation: Beijing experience. HepatoBiliary Surg Nutr 2022;11(3):340-354. doi: 10.21037/hbsn-20-685
36. Dutkowski P, Schlegel A, Slankamenac K, et al. The use of fatty liver grafts in modern allocation systems: risk assessment by the balance of risk (BAR) score. Ann Surg 2012;256:861-8; discussion 868-9. 